\title{
Economic Considerations in MR Imaging of Patients with Cardiac Devices
}

S everal recent high-profile publications have generated interest in MR imaging in patients with implantable cardiac devices, some of which were traditionally considered an absolute contraindication. In particular, an analysis published in the New England Journal of Medicine documented an overall safe experience performing 1500 nonthoracic, 1.5T MRIs in patients with nonconditional devices (1000 pacemakers and 500 implantable cardioverter-defibrillator studies) as part of a prospective registry (The MagnaSafe Registry; http://magnasafe.org/). ${ }^{1}$ Given the wide dissemination of this article, coupled with the estimated 1.8 million patients with similar devices in the United States alone, ${ }^{2}$ practical guidance for radiologists, who are on the front line of this decision-making process, is desperately needed.

In this setting, we wish to express our gratitude to Korutz et al ${ }^{3}$ for their recent article, "Pacemakers in MRI for the Neuroradiologist." Their article includes a thorough assessment of safety considerations in performing MR imaging in patients with implantable cardiac devices. In addition to a detailed review of the recent literature, the authors provide their own multidisciplinary protocol as a helpful reference for radiologists who have instituted (or are considering implementing) MR imaging in this patient population. Furthermore, their own experience imaging 121 patients with nonconditional implants adds to the growing evidence that MR imaging can be performed in appropriately screened and carefully monitored patients.

One additional consideration, separate from any safety issue, must be noted. At present, the Centers for Medicare and Medicaid does not permit coverage for routine clinical MR imaging in patients with cardiac devices that are classified by the FDA as nonconditional. In fact, reimbursement is only permitted when imaging is performed within the narrow scope of a prospective registry assessing safety. ${ }^{4}$ Thus, for most of these patients, the cost of clinically indicated MRIs will not be covered. In our experience, many providers are unaware of this crucial issue. When informed, they appreciate the opportunity to discuss the implications with their patients and, when appropriate, may reconsider using a cov- ered alternate technique. For radiologists, accurate reporting of the specific device and FDA category is essential to ensure remuneration for covered MRI-conditional devices and to avoid fraudulent billing for noncovered services.

Some institutions have elected to perform these studies regardless of the patient's ability to pay an out-of-pocket expense. However, this may not be feasible or desirable for other facilities, given the time-intensive and resource-consuming processes needed to screen these patients and coordinate their imaging. If a self-payment is expected, this should be clearly communicated and prospectively discussed with the patient, who may need to complete an Advance Beneficiary Notice. In certain situations, meeting with a hospital financial representative might help to minimize the impact of a large and unexpected bill following the patient's study.

While the financial considerations entail additional logistic considerations in an already complex process, a proactive approach can ensure financial informed consent and may refine decisions regarding clinical work-up.

\section{REFERENCES}

1. Russo RJ, Costa HS, Silva PD, et al. Assessing the risks associated with MRI in patients with a pacemaker or defibrillator. NEngl J Med 2017; 376:755-64 CrossRef Medline

2. Nazarian S, Hansford R, Roguin A, et al. A prospective evaluation of a protocol for magnetic resonance imaging of patients with implanted cardiac devices. Ann Intern Med 2011;155:415-24 CrossRef Medline

3. Korutz AW, Obajuluwa A, Lester MS, et al. Pacemakers in MRI for the neuroradiologist. AJNR Am J Neuroradiol 2017 Jul 13. [Epub ahead of print] CrossRef Medline

4. Centers for Medicare \& Medicaid Services. Decision Memo for Magnetic Resonance Imaging (MRI) (CAG-00399R2). February 24, 2011. https:// www.cms.gov/medicare-coverage-database/details/nca-decisionmemo.aspx $?$ NCAId $=246 \& b c=$ ACAAAAAAAgAAAA $\% 3 d \% 3 d \& . \quad$ Accessed August 30, 2017

\footnotetext{
(1) M.U. Antonucci (1) M.V. Spampinato (C).R. Ritenour
} Department of Radiology and Radiological Science Medical University of South Carolina Charleston, South Carolina 